\title{
X-Shaped Radio Galaxies and the Nanohertz Gravitational Wave Background
}

\author{
David H. Roberts ${ }^{1}$, Lakshmi Saripalli ${ }^{2}$ and Ravi Subrahmanyan ${ }^{2}$ \\ ${ }^{1}$ Brandeis University \\ email: roberts@brandeis.edu \\ ${ }^{2}$ Raman Research Institute
}

\begin{abstract}
Coalescence of supermassive black holes (SMBHs) in galaxy mergers is potentially the dominant contributor to the low frequency gravitational wave background (GWB). It was proposed by Merritt \& Ekers that X-shaped radio galaxies are signposts of such coalescences and that their abundance might be used to predict the magnitude of the GWB. Cheung identified a sample of 100 candidate X-shaped radio galaxies using the NRAO FIRST survey; these are small-axial-ratio extended radio sources with off-axis emission. In Roberts et al. we made radio images of 52 of these sources with resolution of about 1 arcsecond using archival Very Large Array data. Fifty-one of the 52 were observed at $1.4 \mathrm{GHz}$, seven were observed at 1.4 and $5 \mathrm{GHz}$, and one was observed only at $5 \mathrm{GHz}$. Our higher resolution VLA images along with FIRST survey images of the sources in the sample reveal that extended extragalactic radio sources with small axial ratios are largely $(60 \%)$ cases of double radio sources with twin lobes that have off-axis extensions, usually with inversion-symmetric structure. The available radio images indicate that at most $20 \%$ of sources might be genuine X-shaped radio sources that could have formed by a restarting of beams in a new direction following an interruption and axis flip. The remaining $20 \%$ are in neither of these categories.

These images indicate that at most a small fraction of the candidates might be genuine $\mathrm{X}$ shaped radio sources that were formed by a restarting of beams in a new direction following a major merger, or by spin drift caused by BH-BH interaction. This suggests that fewer than $1.3 \%$ of extended radio sources appear to be candidates for genuine axis reorientations ("spin flips"), or $2.2 \%$ if possible "axis drift" sources are included, much smaller than the $7 \%$ suggested by Leahy \& Parma. Thus, the associated GWB may be substantially smaller than previous estimates. These results can be used to normalize detailed calculations of the SMBH coalescence rate and the GWB.
\end{abstract}

Keywords. gravitational wave background, radio galaxies, supermassive black holes 

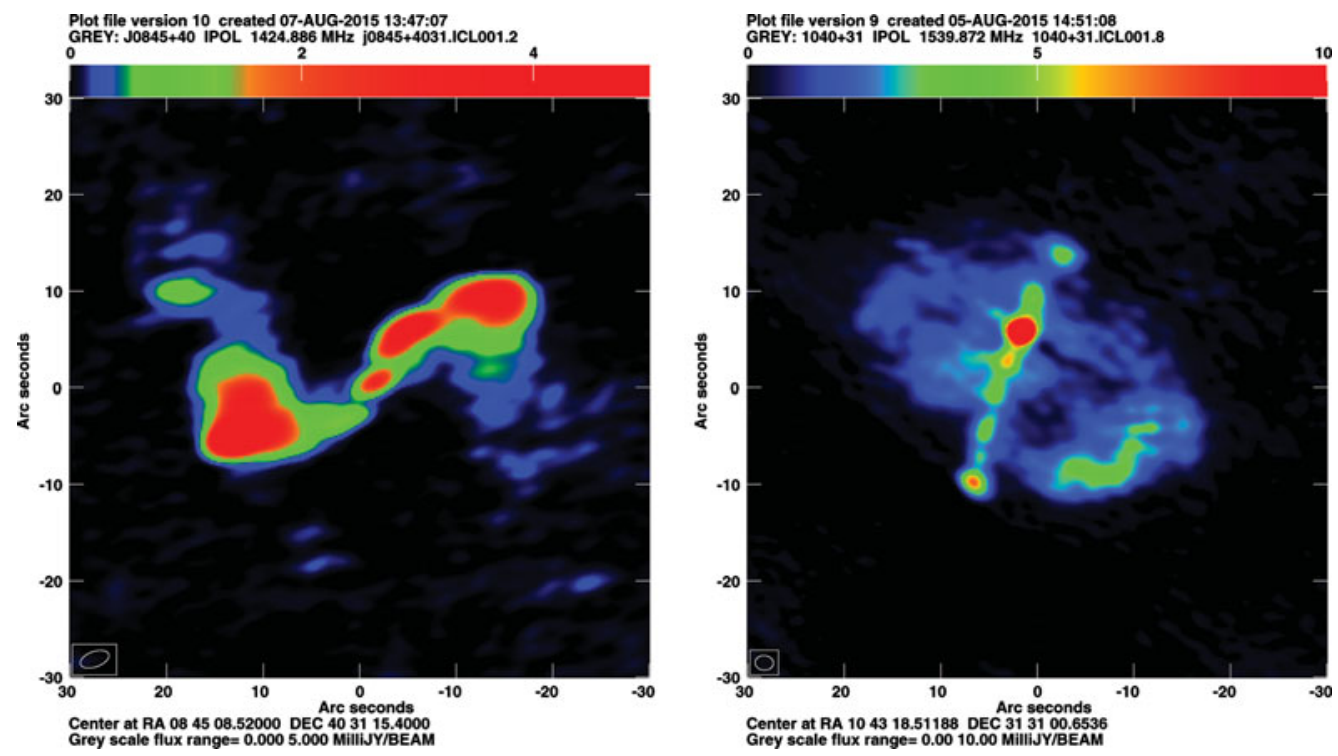

Figure 1. Two examples of $\mathrm{X}$-shaped radio galaxies that may each harbor a pair of supermassive black holes. (left) J0845+4031, a possible axis drift source. (right) J1043+3131, a possible spin flip source.

\section{References}

Cheung, C. C. 2007, AJ, 133, 2097

Leahy, J. P. \& Parma, P. 1992, "Multiple Outbursts in Radio Galaxies," in Extragalactic Radio Sources. From Beams to Jets, ed. J. Roland (Cambridge: Cambridge Univ. Press) p. 307 Merritt, D. \& Ekers, R. D. 2002, Science, 297, 1310

Roberts, D. H., Cohen, J. P., Lu, J., Saripalli, L., \& Subrahmanyan, R. 2015, ApJS, 220, 7 